\title{
Requirements of the Pathologist to the Endoscopist (Biopsy Sampling)
}

\author{
Christian Bussmann \\ Pathologie Viollier, Basel, Switzerland
}

\begin{abstract}
Key Words
Eosinophilic esophagitis · Number of biopsies - Location of biopsy samples · Quality of the biopsy specimen · Cytology
\end{abstract}

\begin{abstract}
The diagnosis of eosinophilic esophagitis is based upon corresponding histological and clinical findings. To obtain a reliable diagnosis, the clinician should ideally submit four biopsies each of the proximal and distal esophagus, respectively. The biopsies should not only encompass the full thickness of the epithelium, but parts of the lamina propria as well.

(c) 2014 S. Karger AG, Basel
\end{abstract}

\section{Introduction}

In the consensus definition of 2011 the diagnosis of eosinophilic esophagitis (EoE) is based upon the sum of clinical and histological findings [1]. The histological hallmark of EoE is a dense eosinophilic infiltrate in the squamous epithelium. However, the presence of such an infiltrate cannot be equated with the diagnosis of EoE as eosinophilic inflammation may be encountered in several other disorders, e.g. gastroesophageal reflux disease (GERD) and achalasia. If the clinical findings fit the diagnosis of EoE, the histological confirmation is usually straightforward. If only a little inflammation is present,

\section{KARGER}

(c) 2014 S. Karger AG, Basel

0257-2753/14/0322-0074\$39.50/0

E-Mail karger@karger.com

www.karger.com/ddi however, the diagnosis may be hampered. Conveyance of all the pertinent clinical information and optimization of biopsy sampling may improve the diagnostic yield in these cases. Thus, close cooperation between clinicians and pathologists is an absolute necessity.

In the following sections we will discuss biopsy protocols that can improve diagnostic efficacy. To optimize biopsy samples provided to the pathologist, three main concerns have to be addressed: (1) the number of biopsies, (2) the location of biopsies, and (3) the size and depth of biopsies.

\section{Number of Biopsies}

The esophagus measures about $25 \mathrm{~cm}$ in length and $5 \mathrm{~cm}$ in diameter, thus expanding over the considerable area of $125 \mathrm{~cm}^{2}$ or $125,000 \mathrm{~mm}^{2}$. A biopsy averages around $4 \times 1 \mathrm{~mm}$ in size, only a fraction of which is examined by histology. Thus, the size of a biopsy represents only a minute part of the esophagus of around $0.002 \%$. The question arises whether such a small tissue sample can be representative, especially considering the frequent patchy distribution of EoE across the esophagus.

The following case illuminates these concerns: a 17-year-old patient underwent endoscopy due to clinical suspicion of EoE. A total of 8 biopsy samples were obtained, 4 each from the distal and the proximal esophagus. Histological examination of all 8 biopsy samples 
Table 1. Comparison of the histological features of GERD and EoE

\begin{tabular}{ll}
\hline EoE & GERD \\
\hline Patchy distribution of the inflammatory infiltrate & $\begin{array}{c}\text { Inflammatory infiltrate exhibits a decreasing gradient from } \\
\text { GEJ to the proximal esophagus } \\
\text { Eosinophils intraepithelial }\left(\geq 15 / \mathrm{HPF} \text { or } \geq 120 / \mathrm{mm}^{2}\right)\end{array}$ \\
$\begin{array}{c}\text { Eosinophils intraepithelial } \\
\text { Eosinophils preferentially located superficial }\end{array}$ & Few eosinophils randomly located (rather in the stroma) \\
Detritic squamous cells and eosinophils on surface & No abscesses \\
Degranulation of eosinophils prominent & No detritus \\
Stromal papillae & No or few degranulation \\
Number reduced & Stromal papillae \\
Narrowed, pointed at the top & Number elevated \\
No or very few lymphocytes & Thickened, especially at the top \\
Capillaries not dilated & Contain lymphocytes \\
Epithelium & Contain dilated capillaries \\
Thickening of basal layer: $+\rightarrow+++$ (up to $90-100 \%)$ & Epithelium \\
No acanthosis (often thin because of smaller basal cells) & Thickening of basal layer: $+\rightarrow+/++$ \\
Spongiosis: $+\rightarrow++$ & Acanthosis: frequent \\
Parakeratosis: sometimes & Spongiosis: no or few \\
Orthokeratosis and no epidermization & Parakeratosis: rare \\
No erosions and no spontaneous ulcers (except Boerhaave's) & Orthokeratosis, sometimes with epidermization: rare \\
Adjacent gastric mucosa: no changes & Ulcers and/or erosions \\
No Barrett's mucosa & Adjacent gastric mucosa \\
& With inflammation as in the esophagus \\
& Sometimes pseudopancreatic metaplasia \\
& Barrett's mucosa can be present \\
\hline
\end{tabular}

$\mathrm{HPF}=$ High-power field; GEJ = gastroesophageal junction .

was inconspicuous. Due to the persistence of symptoms, a repeat endoscopy was performed after 3 months. Biopsies were obtained as indicated above with 7 biopsy samples showing normal histology. However, one biopsy sample now exhibited histological findings consistent with EoE. This example highlights the succinct patchy nature of EoE. The transition of mucosal areas exhibiting normal histology to mucosal areas exhibiting eosinophilic inflammation is known to be frequently abrupt [2].

The question arises of whether targeted biopsies can improve the yield in the diagnosis of EoE. The reliability of endoscopy in the diagnosis of EoE is confounded, however, by the multitude of endoscopic findings in EoE and the frequent divergence of endoscopic and histological findings. Eosinophilic inflammation may be encountered upon histological examination despite an inconspicuous endoscopy result and vice versa. For these reasons, targeted biopsies are not an option to improve diagnostic accuracy and endoscopy results were not included in the consensus definition of EoE [1].

Considering the relation between the size of the esophagus and the size of a biopsy sample, the patchy distribu- tion of EoE and the diagnostic inaccuracy of endoscopy, recommendations have been issued to perform multiple $(\geq 8)$ biopsies.

\section{Location of Biopsies}

Different disorders can cause eosinophilic inflammation with EoE being most frequently at its root. The second most frequent disorder causing eosinophilic inflammation is GERD, which occurs much more commonly than EoE and is by no means always associated with eosinophilic infiltration of the mucosa. However, the diagnosis of GERD always has to be taken into consideration, especially in patients exhibiting ambiguous symptoms.

The histological hallmarks of EoE and GERD are summarized in table 1. Briefly, GERD and EoE share many histological features and differ from each other only slightly. EoE is marked by a more pronounced basal cell hyperplasia and a distinct spongiosis (intercellular edema). In contrast, squamous hyperplasia is usually prominent in GERD and lacking in EoE. Whereas the thickness 
Fig. 1. Comparison of the dimensions of a biopsy and the whole esophagus wall (hematoxylin and eosin histology).

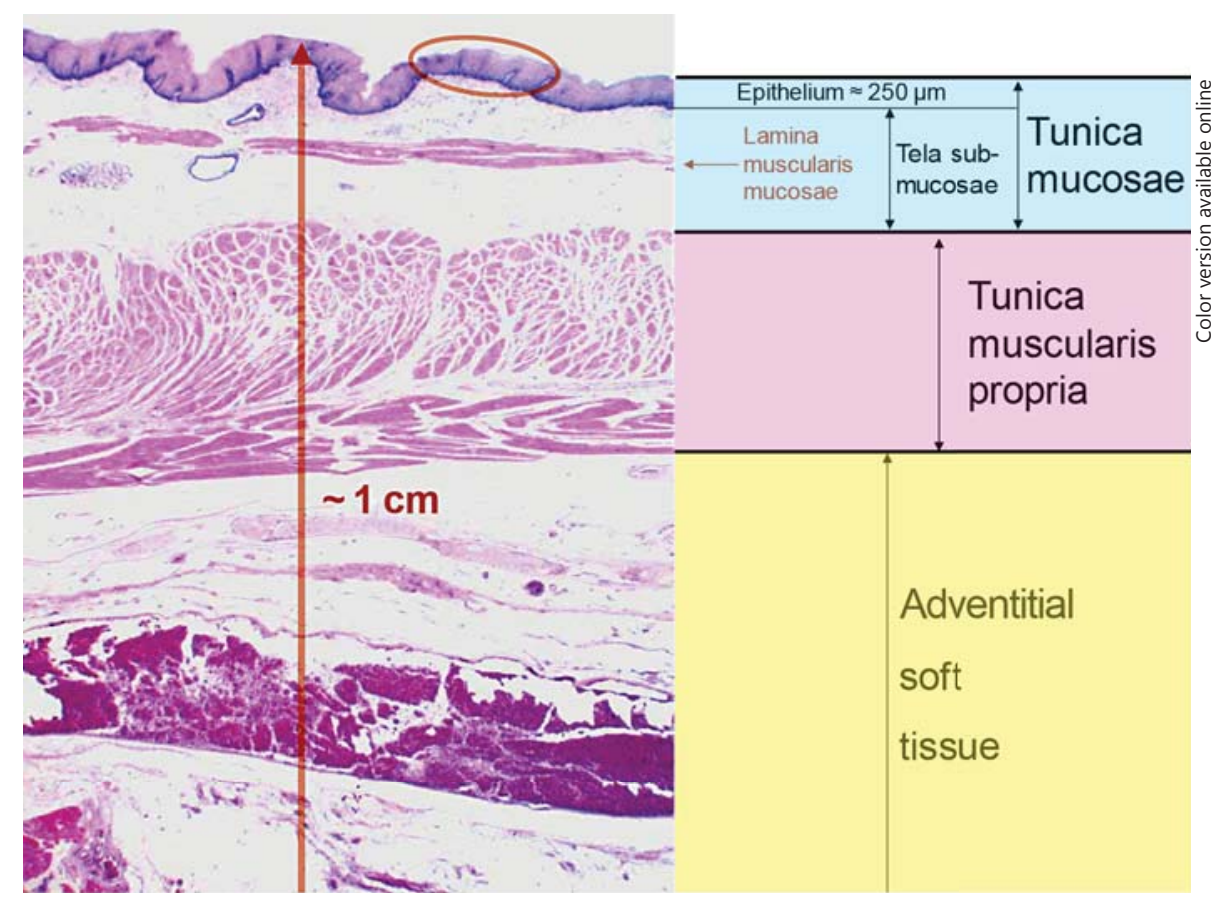

of the normal squamous epithelium measures around 250 $\mu \mathrm{m}$, its width may be more than doubled in GERD. The most important distinction between GERD and EoE lies in the distribution of the inflammatory changes. These display a distinct gradient in GERD, where they are most pronounced in proximity to the gastroesophageal junction and continuously decrease in extent towards the proximal esophagus. In contrast, inflammatory changes are distributed in a patchy fashion throughout the esophagus in EoE as outlined above. Due to the different spatial distribution of the inflammatory infiltrate and considering the aforementioned minimal number of biopsies required, $\geq 4$ biopsies should be obtained from both the distal esophagus, close to the gastroesophageal junction, and the proximal esophagus. These biopsy samples should be submitted in separate vials, each containing $4 \%$ formaldehyde (10\% neutral-buffered formalin).

\section{Depth of the Biopsies}

It is technically more challenging to obtain adequate biopsies from the esophagus compared to other parts of the GI tract. The border between the squamous epithelium and the underlying soft tissue is fragile and thus the squamous epithelium easily detaches. For these reasons, biopsies obtained are frequently small and en- compass only superficial parts of the squamous epithelium. The underlying soft tissue is not sampled at all or only in very minute fractions. It would be of great interest, however, to study the effects of EoE on deeper layers of the esophagus. So far, this issue has only been addressed by one case report, which described a patient with EoE who required an esophageal resection following Boerhaave's syndrome due to food impaction [3]. The histology revealed marked hypertrophy of the muscularis mucosae, fibrosis of the entire submucosa and an eosinophilic infiltrate extending through all esophageal layers into the adventitia. It remains to be seen whether these findings can be generalized to other patients with EoE. All other studies examined minute amounts of lamina propria attached to the squamous epithelium, which raised the question of representative sampling of deeper layers. To obtain a more representative sample, the performance of deeper biopsies encompassing the squamous epithelium and the lamina propria is recommended. The danger of esophageal perforation is minimal if deeper biopsies are obtained, as long as no other esophageal pathology, e.g. ulcerations, is present. The relationship between the full thickness of the esophageal wall of around $10 \mathrm{~mm}$ and the depth of a biopsy of $0.5-1 \mathrm{~mm}$ illustrates the feasibility of this approach (fig. 1). Ultimately, the exact number, location and size of the biopsies obtained have to be adjust- 
ed to individual patients in view of all clinical findings. The aforementioned recommendations cannot be applied to all patients.

\section{Cytology in the Diagnosis of EoE}

To obtain adequate biopsies is difficult and patients have to be sedated. Thus, less invasive procedures such as endoscopy combined with brush biopsies and examination by cytology have been discussed. This approach is not well developed in the assessment of esophageal diseases and is presently only rarely applied in the diagnosis of candida esophagitis, which can easily be diagnosed by cytology. However, endoscopy still has to be performed to obtain specimens for cytological examination.

Experiences with patients suffering from allergic asthma show the efficacy of cytology of the respiratory tract in evaluating these individuals. These data raise hope that cytology may aid in future in the diagnosis of patients with EoE. Although the diagnostic utility of cytology in the diagnosis of EoE is limited, this method may serve as an adjunct in certain patient subsets, especially pediatric patients. A project addressing this issue is planned by the Swiss EoE Group.

\section{Summary}

EoE is defined by clinical and histological findings. Thus, the diagnosis of EoE requires a close cooperation between involved clinicians and pathologists. The pertinent clinical information has to be provided and adequate biopsies have to be obtained. The gastroenterologist should consider the following recommendations when obtaining biopsies:

- Obtain $\geq 8$ biopsies

- Obtain $\geq 4$ biopsies from both the proximal and the distal esophagus, and submit these in two separate vials each containing $4 \%$ formaldehyde ( $10 \%$ neutralbuffered formalin)

- Depending on the clinical question, collect deeper biopsies encompassing squamous epithelium and lamina propria

The role of cytology in the diagnosis of EoE remains to be assessed.

\section{Disclosure Statement}

The author declares that no financial or other conflict of interest exists in relation to the content of the article.

\section{References}

Liacouras CA, Furuta GT, Hirano I, et al: Eosinophilic esophagitis: updated consensus recommendations for children and adults. J Allergy Clin Immunol 2011;128:3-20.

2 Gonsalves N, Policarpio-Nicolas M, Zhang Q, et al: Histopathologic variability and endoscopic correlates in adults with eosinophilic esophagitis. Gastrointest Endosc 2006;64: 313-319.
Fontillon M, Lucendo AJ: Transmural eosinophilic infiltration and fibrosis in a patient with non-traumatic Boerhaave's syndrome due to eosinophilic esophagitis. Am J Gastroenterol 2012;107:1762. 